DOI: 10.2478/awutm-2013-0014

年

VERSITA
Analele Universităţii de Vest,

Timişoara

Seria Matematică - Informatică

LI, 2, (2013), 39- 46

\title{
On the Randic and Sum-Connectivity Index of Nanotubes
}

Mohammad Reza Farahani

\begin{abstract}
Milan Randić proposed in 1975 a structural descriptor called the branching index that later became the well-known Randić connectivity index; it is defined on the ground of vertex degrees $\chi(G)=\sum_{e=u v \in E(G)} \frac{1}{\sqrt{d_{u} d_{v}}}$. In 2008, B. Zhou and $N$. Trinajstić proposed another connectivity index, named the Sumconnectivity index $X(G)$.In this paper, we focus on the structure of $" G=V C_{5} C_{7}[p, q] "$ and " $H=H C_{5} C_{7}[p, q] "$ nanotubes and counting Randić index $\chi(G)=\sum_{e=u v \in E(G)} \frac{1}{\sqrt{d_{u} d_{v}}}$ and sum-connectivity index $X(G)=\sum_{v_{u} v_{v}} \frac{1}{\sqrt{d_{u}+d_{v}}}$ of these nanotubes.
\end{abstract}

AMS Subject Classification (2010). Primary 05C12; secondary 05A15

Keywords. Nanotubes; Randić index; Sum-Connectivity index.

\section{Introduction}

Let $G=(V ; E)$ be a simple connected graph. The sets of vertices and edges of $G$ are denoted by $V=V(G)$ and $E=E(G)$, respectively. A molecular graph is a simple graph such that its vertices correspond to the atoms and the edges to the bonds.

In graph theory, we have many different connectivity topological index of an arbitrary graph $G$. A topological index is a numeric quantity from the structural graph of a molecule which is invariant under graph automorphisms. 
The simplest topological indices are the number of vertices, the number of edges and degree of a vertex $v$ of the graph $G$ and we denoted by $n, e$ and $d_{v}$, respectively. The degree of a vertex is equal to the number of its first neighbors. Also, $\forall u, v \in V(G)$, the distance $d(u, v)$ between $u$ and $v$ is defined as the length of any shortest path in $G$ connecting $u$ and $v$.

The connectivity index introduced in 1975 by Milan Randic [12], who has shown this index to reflect molecular branching. Randić index (First connectivity index) was defined as follows

$$
\chi(G)=\sum_{e=u v \in E(G)} \frac{1}{\sqrt{d_{u} d_{v}}}
$$

In general, the m-connectivity index of a graph $G$ is defined as

$$
{ }^{m} \chi(G)=\sum_{v_{i_{1}} v_{i_{2}} \ldots v_{i_{m+1}}} \frac{1}{\sqrt{d_{i_{1}} d_{i_{2}} \ldots d_{i_{m+1}}}},
$$

where $v_{i_{1}} v_{i_{2}} \ldots v_{i_{m+1}}$ runs over all paths of length $m$ in $G$.

Recently, a closely related variant of the Randić connectivity index called the sum-connectivity index was introduced by B. Zhou and N. Trinajstić $[13,16]$ in 2008. For a connected graph $G$, its sum-connectivity index $X(G)$ is defined as the sum over all edges of the graph of the terms $\frac{1}{\sqrt{d_{u}+d_{v}}}$, that is,

$$
X(G)=\sum_{v_{u} v_{v}} \frac{1}{\sqrt{d_{u}+d_{v}}}
$$

where $d_{u}$ and $d_{v}$ are the degrees of the vertices $u$ and $v$, respectively.

In this paper, we focus on the above connectivity indices "Randic" and "sumconnectivity" index and compute two indices for two types of nanotubes ("G $=V C_{5} C_{7}[p, q] "$ and " $H=H C_{5} C_{7}[p, q] "$ ). Our notation is standard and for more information and background biography, refer to paper series [1-18].

\section{Main Result}

The aim of this section is to compute the Randic connectivity index and sumconnectivity index of $G=V C_{5} C_{7}[p, q]$ and $H=H C_{5} C_{7}[p, q]$ nanotubes. The structure of these nanotubes are consist of cycles with length five and seven (or $C_{5} C_{7}$ net) by different compound. A $C_{5} C_{7}$ net is a trivalent decoration made by alternating $C_{5}$ and $C_{7}$. It can cover either a cylinder or a torus. For a review, historical details and further bibliography see the 3-dimensional 
Vol. LI (2013) On the Randic and Sum-Connectivity Index...



(B)

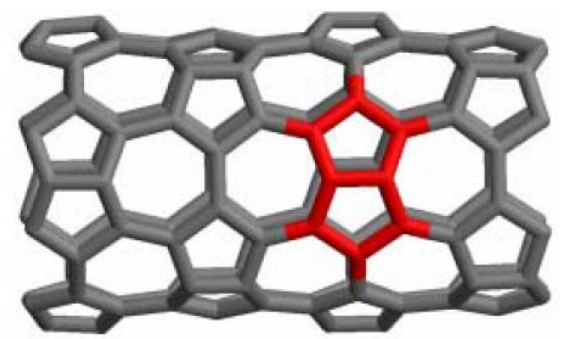

(A)

Figure 1: The Molecular graph of $V C_{5} C_{7}(\mathrm{~A})$ and $H C_{5} C_{7}(\mathrm{~B})$ nanotube.

lattice of $V C_{5} C_{7}[p, q]$ and $H C_{5} C_{7}[p, q]$ nanotubes in Figure 1 and their 2dimensional lattice in Figure 2 and Figure 3, respectively.

Before presenting the main results, let us introduce some definitions. First, let us denote the number of pentagons in the first row of the 2D-lattice of $G$ (Figure 2) and $H$ (Figure 3) by $p$. In these nanotubes, the four first rows of vertices and edges are repeated alternatively, we denote the number of this repetition by $q . \forall p, q \in \mathbb{N}$ in each period of $G=V C_{5} C_{7}[p, q]$, there are $16 p$ vertices and $6 p$ vertices which are joined to the end of the graph. Thus the number of vertices in $G$ is equal to

$$
n=\left|V\left(V C_{5} C_{7}[p, q]\right)\right|=16 p q+6 p .
$$

Since $3 p+3 p$ vertices have degree two and other have degree three $(16 p q)$, thus the number of edges in this nanotube is equal to

$$
e=\left|E\left(V C_{5} C_{7}[p, q]\right)\right|=\frac{2(6 p)+3(16 p q)}{2}=24 p q+6 p .
$$

Also, in each period of $H=H C_{5} C_{7}[p, q]$, there are $8 p$ vertices. Hence

$$
n=\left|V\left(H C_{5} C_{7}[p, q]\right)\right|=8 p q+5 p,
$$

$5 p$ vertices which are joined to the end of $H$. And in each period there are $12 p$ edges and we have $q$ repetition and $5 p$ addition edges, thus the number of edges in this nanotube is equal to $e=\left|E\left(H C_{5} C_{7}[p, q]\right)\right|=12 p q+5 p$, $\forall p, q \in \mathbb{N}$. On the other hands $2 p+3 p$ vertices have degree two and $8 p q$ other vertices have degree three, and alternatively

$$
e=\frac{2(5 p)+3(8 p q)}{2}=12 p q+5 p
$$

Definition 2.1. Let $G=(V ; E)$ be a simple connected graph and $d_{v}$ is degree of vertex $v \in V(G)$ (Obviously $1 \leq \delta \leq d_{v} \leq \Delta \leq n-1$, such that 
$\delta=\operatorname{Min}\left\{d_{v} \mid v \in V(G)\right\}$ and $\left.\Delta=\operatorname{Max}\left\{d_{v} \mid v \in V(G)\right\}\right)$. We divide edge set $E(G)$ and vertex set $V(G)$ of graph $G$ to several partitions, as follow:

$$
\begin{array}{r}
\forall i, 2 \delta \leq i \leq 2 \Delta, E_{i}=\left\{e=u v \in E(G) \mid d_{v}+d_{u}=i\right\}, \\
\forall j, \delta^{2} \leq j \leq \Delta^{2}, E_{j}^{*}=\left\{e=u v \in E(G) \mid d_{v} \times d_{u}=j\right\} \\
\text { and } \quad \forall k, \delta \leq k \leq \Delta, V_{k}=\left\{v \in V(G) \mid d_{v}=k\right\} .
\end{array}
$$

Obviously, in nano science an atom (or a vertex $v$ ) of a nano structure $G$ have at most four adjacent. In other words, $d_{v}$ is equal to $1,2,3$ and 4 . Therefore, we have two partitions

- $V_{3}=\left\{v \in V(G) \mid d_{v}=3\right\}$

- $V_{2}=\left\{v \in V(G) \mid d_{v}=2\right\}$.

Note that hydrogen and single carbon atoms are often omitted. Also, the edge set of a molecular graph $G$ can be dividing to three partitions, e.g. $E_{4}$, $E_{5}$ and $E_{6}$. In other words,

- For every $e=u v$ belong to $E_{4}, d_{u}=d_{v}=2$.

- Similarly, for every $e=u v$ belong to $E_{6}, d_{u}=d_{v}=3$.

- Finally, for every $e=u v$ belong to $E_{5}$, then $d_{u}=2$ and $d_{v}=3$.

Now, we have following theorems.

Theorem 2.2. Let $G$ be $V C_{5} C_{7}[p, q]$ nanotubes. Then:

- Randić connectivity index of $G$ is equal to

$$
\chi\left(V C_{5} C_{7}[p, q]\right)=8 p q+2(\sqrt{6}-1) p .
$$

- sum-connectivity index of $G$ is equal to

$$
X\left(V C_{5} C_{7}[p, q]\right)=4 \sqrt{6} p q+\left(\frac{12 \sqrt{5}}{5}-\sqrt{6}\right) p
$$

Proof. $\forall p, q \in \mathbb{N}$ consider nanotubes $G=V C_{5} C_{7}[p, q]$ with $16 p q+6 p$ vertices and $24 p q+6 p$ edges, such that $\left|V_{2}\right|=6 p$ and $\left|V_{3}\right|=16 p q$. So, we mark the edges of $E_{5}, E_{6}^{*}$ by red color and the edges of $E_{6}, E_{9}^{*}$ by black color (Figure 2). Thus, we have

- $\left|E_{5}\right|=\left|E_{6}^{*}\right|=6 p+6 p$

- $\left|E_{6}\right|=\left|E_{9}^{*}\right|=24 p q-6 p$. 
Now, by according to definition of Randić connectivity index

$$
\begin{aligned}
{ }^{1} \chi\left(V C_{5} C_{7}[p, q]\right)= & \sum_{e=u v \in E(G)} \frac{1}{\sqrt{d_{u} d_{v}}}=\sum_{e=u v \in E_{9}^{*}} \frac{1}{\sqrt{d_{u} d_{v}}}+\sum_{e=u v \in E_{6}^{*}} \frac{1}{\sqrt{d_{u} d_{v}}} \\
& =\frac{\left|E_{9}^{*}\right|}{\sqrt{9}}+\frac{\left|E_{6}^{*}\right|}{\sqrt{6}} \\
& =\frac{24 p q-6 p}{\sqrt{9}}+\frac{12 p}{\sqrt{6}} \\
& =8 p q+2(\sqrt{6}-1) p .
\end{aligned}
$$

Also, by according to the definition of sum-connectivity index, we have following equations:

$$
\begin{aligned}
& { }^{1} X\left(V C_{5} C_{7}[p, q]\right)=\sum_{e=u v \in E(G)} \frac{1}{\sqrt{d_{u}+d_{v}}}=\sum_{e=u v \in E_{6}} \frac{1}{\sqrt{d_{u}+d_{v}}}+\sum_{e=u v \in E_{5}} \frac{1}{\sqrt{d_{u}+d_{v}}} \\
& =\frac{\left|E_{6}\right|}{\sqrt{6}}+\frac{\left|E_{5}\right|}{\sqrt{5}} \\
& =\frac{24 p q-6 p}{\sqrt{6}}+\frac{12 p}{\sqrt{5}} \\
& =4 \sqrt{6} p q+\left(\frac{12 \sqrt{5}}{5}-\sqrt{6}\right) p \text {. }
\end{aligned}
$$
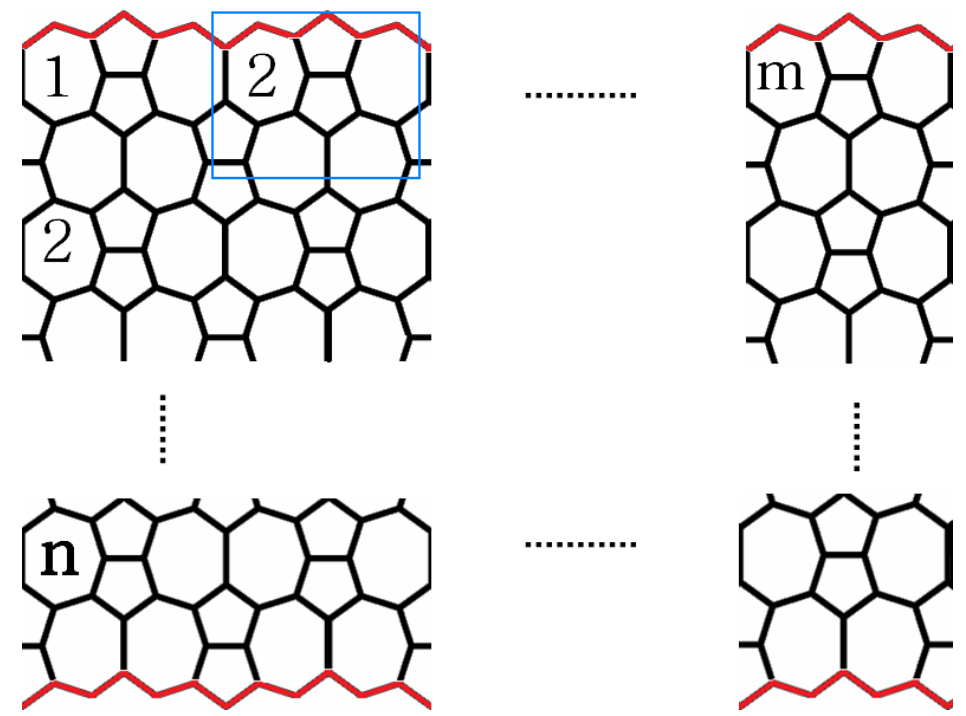

Figure 2: 2-Dimensional Lattice of $G=V C_{5} C_{7}[m, n]$.

Here, we complete the proof of Theorem 2.2. 

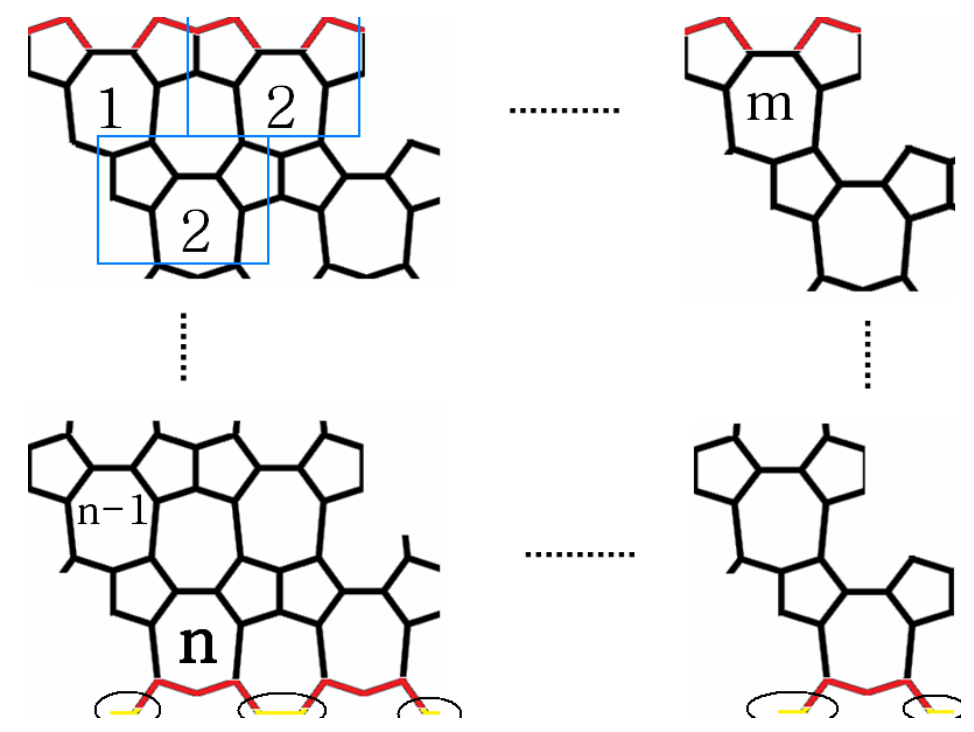

Figure 3: 2-Dimensional Lattice of $H=H C_{5} C_{7}[m, n]$.

Theorem 2.3. $\forall p, q \in \mathbb{N}$

- Randić connectivity index of $\mathrm{HC}_{5} C_{7}[p, q]$ nanotube is equal to

$$
\chi\left(H C_{5} C_{7}[p, q]\right)=4 p q+\left(\frac{8 \sqrt{6}-5}{6}\right) p .
$$

- Sum-connectivity index of $\mathrm{HC}_{5} \mathrm{C}_{7}[p, q]$ nanotube is equal to

$$
X\left(H C_{5} C_{7}[p, q]\right)=2 \sqrt{6} p q+\left(\frac{8 \sqrt{5}}{5}-\frac{2 \sqrt{6}}{3}+\frac{1}{2}\right) p
$$

Proof. Consider nanotube $H=H C_{5} C_{7}[p, q], \forall p, q \in \mathbb{N}$. Similar to $V C_{5} C_{7}[p, q]$ nanotube, $H$ consists of heptagon and pentagon nets. But, in this nanotube there are $8 p q+5 p$ atoms (vertices) and $12 p q+5 p$ bonds (edges). Such that $\left|V_{2}\right|=5 p$ and $\left|V_{3}\right|=8 p q$, and alternatively $\bullet\left|E_{4}\right|=\left|E_{4}^{*}\right|=p$

- $\left|E_{5}\right|=\left|E_{6}^{*}\right|=4 p+4 p$

- $\left|E_{6}\right|=\left|E_{9}^{*}\right|=12 p q-4 p$.

We mark all edge $E_{4}, E_{5}$ and $E_{6}$ by yellow, red and black color in Figure 3, respectively. 
Thus, we have following equations for its connectivity indies.

$$
\begin{aligned}
& { }^{1} \chi\left(H C_{5} C_{7}[p, q]\right)=\sum_{e=u v \in E(H)} \frac{1}{\sqrt{d_{u} d_{v}}} \\
& =\sum_{e=u v \in E_{9}^{*}} \frac{1}{\sqrt{d_{u} d_{v}}}+\sum_{e=u v \in E_{6}^{*}} \frac{1}{\sqrt{d_{u} d_{v}}}+\sum_{e=u v \in E_{4}^{*}} \frac{1}{\sqrt{d_{u} d_{v}}} \\
& =\frac{\left|E_{9}^{*}\right|}{\sqrt{9}}+\frac{\left|E_{6}^{*}\right|}{\sqrt{6}}+\frac{\left|E_{4}^{*}\right|}{\sqrt{4}}=\frac{12 p q-4 p}{3}+\frac{8 \sqrt{6} p}{6}+\frac{p}{2} \\
& =4 p q+\left(\frac{8 \sqrt{6}-5}{6}\right) p \text {. } \\
& { }^{1} X\left(H C_{5} C_{7}[p, q]\right)=\sum_{e=u v \in E(H)} \frac{1}{\sqrt{d_{u} d_{v}}} \\
& =\sum_{e=u v \in E_{6}} \frac{1}{\sqrt{d_{u} d_{v}}}+\sum_{e=u v \in E_{5}} \frac{1}{\sqrt{d_{u} d_{v}}}+\sum_{e=u v \in E_{4}} \frac{1}{\sqrt{d_{u} d_{v}}} \\
& =\frac{\left|E_{6}\right|}{\sqrt{6}}+\frac{\left|E_{5}\right|}{\sqrt{5}}+\frac{\left|E_{4}\right|}{\sqrt{4}}=\frac{12 p q-4 p}{\sqrt{6}}+\frac{8 p}{\sqrt{5}}+\frac{p}{2} \\
& =2 \sqrt{6} p q+\left(\frac{8 \sqrt{5}}{5}-\frac{2 \sqrt{6}}{3}+\frac{1}{2}\right) p \text {. }
\end{aligned}
$$

And these complete the proof of Theorem 2.3.

\section{References}

[1] J. Chen and S. Li, On the sum-connectivity index of unicyclic graphs with k pendent vertices, Math. Commun., 16, (2011), 359-368

[2] Z. Du, B. Zhou, and N. Trinajstić, Minimum sum-connectivity indices of trees and uni-cyclic graphs of a given matching number, J. Math. Chem., 47, (2010), 842855

[3] Z. Du and B. Zhou, On sum-connectivity index of bicyclic graphs, Bull. Malays. Math. Sci. Soc., 35 (1), (2012), 101-117

[4] Z. Du, B. Zhou, and N. Trinajstić, A note on generalized sum-connectivity index, Appl. Math. Lett., 24, (2010), 402-405

[5] M.R. Farahani, Computing Randic, Geometric-Arithmetic and Atom-Bond Connectivity indices of Circumcoronene Series of Benzenoid, Int. J. Chem. Model., 5 (4), (2013) 
[6] M.R. Farahani, Some Connectivity Indices and Zagreb Index of Polyhex Nanotubes, Acta Chim. Slov., 59, (2012), 779-783

[7] M.R. Farahani, Third-Connectivity and Third-sum-Connectivity Indices of Circumcoronene Series of Benzenoid $H_{k}$, Acta Chim. Slov, 60, (2013), 198-202

[8] M.R. Farahani, Second-sum-connectivity index of Capra-designed planar Benzenoid series $\mathrm{Ca}_{n}\left(C_{6}\right)$, Polymers Research Journal, 7 (3), (2013)

[9] M.R. Farahani, Computing some connectivity indices, Zagreb index and Zagreb polynomial of $\mathrm{VC}_{5} \mathrm{C}_{7}[p, q]$ and $\mathrm{HC}_{5} \mathrm{C}_{7}[p, q]$ nanotubes, Chemical Physics Research Journal, 6 (1), (2013)

[10] M.R. Farahani, Computing First-, Second -Connectivity index and First-, SecondSum-Connectivity index of Circumcoronene series of benzenoid, Submitted for publication, (2013)

[11] B. Lucic, S. Nikolic, N. Trinajstić, B. Zhou, and S. Ivanis Turk, SumConnectivity Index, (2010), 101-136

[12] M. Randić, On characterization of molecular branching, J. Amer. Chem. Soc., 97, (1975), 6609

[13] D. Vukićević and B. Furtula, Topological index based on the ratios of geometrical and arithmetical means of end-vertex degrees of edges, J. Math. Chem., 46, (2009), 1369

[14] D. Vukicevic, N. Trinajstić, S. Nikolic, B. Lucic, and B. Zhou, Master Connectivity Index and Master Connectivity Polynomial, Current Computer-Aided Drug Design, 6, (2010), 235-239

[15] R. Xing, B. Zhou, and N. Trinajstićc, Sum-connectivity index of molecular trees, J. Math. Chem., 48, (2001), 583-591

[16] B. Zhou and N. Trinajstić, On a novel connectivity index, J. Math. Chem., 46, (2009), 1252-1270

[17] B. Zhou and N. Trinajstić, On General Sum-Connectivity Index, J. Math. Chem., 47, (2010), 210-218

[18] B. Zhou and N. Trinajstić, Minimun General Sum-Connectivity Index of Unicyclic Graphs, J. Math. Chem., 48, (2010), 697-703

Mohammad Reza Farahani

Department of Applied Mathematics,

Iran University of Science and Technology (IUST),

Narmak, Tehran 16844, Iran

E-mail: MR_Farahani@mathdep.iust.ac.ir \& MRFarahani88@gmail.com

Received: 12.07.2013

Accepted: 2.10 .2013

Revised: 1.11 .2013 